\title{
Resistance to Cyclic Fatigue of Nickel-Titanium Files Immersed in Sodium Hypochlorite at Body Temperature
}

\author{
Hind F. Abuhulaibah and Ammar AbuMostafa \\ Restorative Dentistry Department, Endodontics Division, Riyadh Elm University, Riyadh, Saudi Arabia \\ Correspondence should be addressed to Ammar AbuMostafa; ammarabumostafa@hotmail.com
}

Received 24 July 2020; Revised 6 September 2020; Accepted 8 September 2020; Published 19 September 2020

Academic Editor: Spiros Zinelis

Copyright (c) 2020 Hind F. Abuhulaibah and Ammar AbuMostafa. This is an open access article distributed under the Creative Commons Attribution License, which permits unrestricted use, distribution, and reproduction in any medium, provided the original work is properly cited.

Background/purpose. To assess the resistance to cyclic fatigue (CF) displayed by two nickel-titanium (NiTi) files, One Curve (OC) and ProTaper Gold (PTG), after their immersion in 2.5\% sodium hypochlorite ( $\mathrm{NaOCl}$ ) solution at body temperature. Materials and Methods. Forty-five files from each brand were randomly assigned to three groups $(n=15)$ and subjected to the following: no immersion (control), 1-minute immersion, and 5 -minute immersion in $2.5 \% \mathrm{NaOCl}$ at $37^{\circ} \mathrm{C}$. CF for all the files was tested within a well-lubricated stainless-steel artificial canal in a water bath at $37^{\circ} \mathrm{C}$ simulating body temperature. The procedure was video recorded, and the number of cycles to fracture (NCF) was calculated by multiplying the time taken to fracture, with the number of rotations per second. The data were analyzed for normality, and thereafter, one-way ANOVA with multiple Bonferroni was used as the post hoc test. Results. The resistance to CF of OC files was significantly higher than PTG files in all groups. In groups immersed in $\mathrm{NaOCl}$ for one minute, OC files showed significant drop in the CF resistance; PTG files showed a drop as well but not significantly. Both files demonstrated a significant drop in resistance to CF after immersion in $\mathrm{NaOCl}$ for five minutes compared to the control group. Conclusion. OC files were significantly more resistant to $\mathrm{CF}$ compared to PTG in all groups. Immersion in $2.5 \% \mathrm{NaOCl}$ for 5 minutes significantly reduced the resistance to $\mathrm{CF}$ for all the files.

\section{Introduction}

Present day nickel-titanium (NiTi) rotary files are used for root canal cleaning and shaping mainly because of their superelasticity [1]. NiTi files were introduced during the 1980 s, which offered significant advantages such as the ability to maintain the original root canal shape and prevent the creation of irregularities inside the root canal [2].

Despite these advantages, NiTi files appear to have a risk of separation mainly because of flexural cyclic fatigue and torsional fracture [3]. A study demonstrated that $70 \%$ of fractures were because of cyclic fatigue, while $30 \%$ was torsional fatigue [4]. Researchers in another study evaluated 27 fractured ProTaper files and found flexural fatigue to be the major reason for failure (92.5\%) [5]. Cyclic fatigue failure happens as a result of tension-compression stress cycles in the area of maximum flexure, particularly during preparing curved canals. Torsional fracture occurs when part of the file is locked to the dentin, while the shank maintains to rotate [6].

Corrosion is an additional factor that might limit the resistance to fatigue fracture, and it occurs during root canal cleaning and shaping when files come in contact with sodium hypochlorite $(\mathrm{NaOCl})$ solution. Pits and surface defects are created which act as propagating point for fatigue failure of the files [3]. Effect of $\mathrm{NaOCl}$ on the cyclic fatigue resistance of the NiTi files was investigated through several studies, and findings were controversial [7-13]. Moreover, size, cross section, and core area of the files affect their cyclic fatigue resistance $[14,15]$.

The technology of the manufacture of the NiTi instruments is always being improved. The aim of the improvement is to get the instruments with superior flexibility and better resistance versus cyclic fatigue; not only the 
development of the manufacturing method but also the usage of the new alloys helps to increase the effectiveness and safety of the NiTi instruments. Improvement of the crosssectional design, thermal treatment protocols, electropolishing, and electrodischarge machining (EDM) are some of the preferred methods [16].

ProTaper Gold files (Dentsply Sirona, York, PA) (PTG) were commercially introduced in 2014, geometrically identical to the ProTaper Universal (Dentsply Sirona, York, PA) design, but with enhanced metallurgical properties owing to the heat treatment process of the alloy [17]. It has been demonstrated that the fatigue resistance of ProTaper Gold was higher than ProTaper Universal [18].

Later, in 2018, One Curve files (Micro Mega, Besancon, France) (OC) were produced using a heat-treated C-wire technology. According to the manufacturer, the file has a controlled memory, which can present a better performance in the root canal along with enhanced accessibility [19]. Several studies have investigated the cyclic fatigue resistance of OC files, all of which demonstrated a high resistance to fracture. [20-24].

A literature search revealed that no prior research has evaluated the effects of immersion in sodium hypochlorite on the cyclic fatigue resistance of $\mathrm{OC}$ in comparison with PTG. The aim of the study was to assess the resistance to cyclic fatigue of the two nickel-titanium (NiTi) files, One Curve and ProTaper Gold, after immersion in $\mathrm{NaOCl}$ solution $(2.5 \%)$ at body temperature for varying time intervals. The null hypothesis was that there would be no difference in the cyclic fatigue resistance of the tested files.

\section{Materials and Methods}

Two commercially available NiTi rotary files, namely, OC and PTG, with equal sample distribution $(n=45)$, were used in this study. All the files tested possessed an ISO tip size of 25 and length of $25 \mathrm{~mm}$ and were examined for defects or deformities prior to the experiment, using an operating microscope under $\times 25.6$ magnification (Zumax OMS2350, Dental Microscope); no file was discarded. The 45 files of the same brand were randomly distributed into three groups of 15 each. The first group (control) composed of new files that were not immersed in solution. All files in the second and third groups were immersed in $\mathrm{NaOCl} 2.5 \%$ (Diaa, USA) at $37^{\circ} \mathrm{C}$ to a depth of $16 \mathrm{~mm}$ for 1 and 5 minutes, respectively. The temperature was controlled using a water bath machine (GFL, Germany). Immediately after the immersion, the files were rinsed with bidistilled water at $37^{\circ} \mathrm{C}$ to neutralize the effect of the irrigating solution, dried, and stored in labeled glass containers.

For the purpose of this study, a testing device was made of a stainless-steel block fixed to a main frame. The steel block was machined according to the recommendations of Larsen et al. [25] and Haikel et al. [26] and possessed a simulated root canal, $25 \mathrm{~mm}$ in length, with a single curvature of $60^{\circ}$ and $5 \mathrm{~mm}$ radius that was engraved into the block to a depth of $1.5 \mathrm{~mm}$. The length of the canal was verified by inserting a $K$-file size 15 . The steel block was covered with a removable glass plate to help visualize the file in motion, secure the file in the canal, and allow for the easy retrieval of the broken file fragments.

An ultraslow electric motor handpiece (X-Smart; Dentsply Maillefer), with a gear ratio $16: 1$, was attached to the frame and accurately positioned in a reproducible relationship to the steel block such that each file would be oriented in the middle of the artificial canal, perpendicular to the orifice. Care was taken to ensure that the glass cover was not in contact with the file. A lubrication spray (Pegasus Hand Piece Lubrication Spray, UK) was introduced within the canal space to reduce frictional heat.

Cyclic fatigue for all the rotary files was conducted in a water bath machine (GFL, Germany) filled with water maintained at $37^{\circ} \mathrm{C}$. Test was done under continuous clockwise rotation at the individual manufacturer's recommended speed for each system. The entire process was video recorded, from commencement of rotation, until file fracture was observed, using a mobile camera. Thereafter, the total number of cycles to fracture (NCF) for each file was calculated by multiplying the time (seconds) to fracture by the number of rotations or cycles per second.

The Shapiro-Wilk test of the NCF of the files showed the data to be normally distributed $\left(\mathrm{W}_{(90)}=0.43\right.$, $p=0.234$ ), necessitating the use of parametric statistics. One-way ANOVA with multiple Bonferroni post hoc test was used to compare the significance of difference among the different groups. The level of confidence for all tests was set at $p<0.05$. All data were analyzed using the SPSS ver. 25 data processing software (IBM Corp. Armonk, NY, USA).

\section{Results}

The NCF was compared among the different groups tested and subjected to the one-way ANOVA (Table 1). The oneway ANOVA showed significant differences among the different files tested $(F=33.152, p<0.001)$.

It was observed that the files of the OC group had higher NCF than the files of the PTG group regardless of immersion protocol. The multiple Bonferroni tests showed that the PTG-5-min $\mathrm{NaOCl}$ had significantly lower NCF than all other groups except for PTG-1-min $\mathrm{NaOCl}$. The PTG-1-min NaOCl group had a higher NCF than the PTG-5-min NaOCl and a lower NCF than the PTG-control group. However, these differences were not statistically significant. The PTG-1-min NaOCl group had a significantly lower NCF than the three protocols of the OC group. The PTG-control group had lower NCF than the files of the OC group. While this difference was statistically significant for the OC-control and OC-1-min $\mathrm{NaOCl}$ groups, the difference was not statistically significant when compared to the OC-5-min NaOCl.

The OC-control group had significantly higher NCF than all the other groups in the study. The OC-1-min $\mathrm{NaOCl}$ group had a significantly lower NCF when compared to the OC-control group. Although the NCF of the OC-1-min $\mathrm{NaOCl}$ was higher than the OC-5-min $\mathrm{NaOCl}$ group, the difference was not statistically significant. 
TABLE 1: Comparison of mean number of cycles to fracture (NCF) with post hoc Bonferroni comparisons.

\begin{tabular}{|c|c|c|c|c|c|c|}
\hline \multirow[t]{2}{*}{ Number of cycles to fracture (NCF) } & \multirow[t]{2}{*}{ Mean } & \multirow[t]{2}{*}{ Standard deviation } & \multicolumn{2}{|c|}{$\begin{array}{l}\text { 95\% confidence } \\
\text { interval for mean }\end{array}$} & \multirow[t]{2}{*}{$F^{*}$} & \multirow[t]{2}{*}{$p$} \\
\hline & & & $\mathrm{Lb}$ & $\mathrm{Ub}$ & & \\
\hline PTG-control ${ }^{\mathrm{a}, \mathrm{e}}$ & 752.00 & 165.37 & 660.42 & 843.58 & 33.152 & $p \leq 0.001$ \\
\hline PTG-1-min $\mathrm{NaOCl}^{\mathrm{a}, \mathrm{b}}$ & 651.00 & 165.64 & 559.27 & 742.73 & & \\
\hline PTG-5-min $\mathrm{NaOCl}^{\mathrm{b}}$ & 490.33 & 120.65 & 423.52 & 557.14 & & \\
\hline OC-control $^{\mathrm{C}}$ & 1325.00 & 334.70 & 1139.65 & 1510.35 & & \\
\hline OC-1-min $\mathrm{NaOCl}^{\mathrm{d}}$ & 1038.33 & 204.12 & 925.30 & 1151.37 & & \\
\hline OC-5-min $\mathrm{NaOCl}^{\mathrm{d}, \mathrm{e}}$ & 962.33 & 145.80 & 881.59 & 1043.07 & & \\
\hline
\end{tabular}

${ }^{*}$ Calculated using one-way ANOVA. ${ }^{\mathrm{a}, \mathrm{b}, \mathrm{c}, \mathrm{d}, \mathrm{e}}$ Differences in superscript indicate significant $(p<0.05)$ differences between groups.

\section{Discussion}

The present study compared the cyclic fatigue resistance of two NiTi files, OC with PTG, after immersion in $2.5 \%$ $\mathrm{NaOCl}$ for one and five minutes at $37^{\circ} \mathrm{C}$, followed by cyclic fatigue testing which was also done at $37^{\circ} \mathrm{C}$ in a water bath. The significance of conducting both the immersion and testing at body temperature was to mimic the clinical condition and thereby obtain dependable data [11,27]. Care was also taken to immerse only $16 \mathrm{~mm}$ from the tip of files in the solution, to avoid galvanic corrosion phenomena [12].

The overall inference obtained from the results of this study is that OC is significantly more resistant to cyclic fatigue failure compared to PTG, thereby refuting the null hypothesis.

Although there have been no prior research done comparing these particular brands of NiTi files for their resistant to cyclic fatigue, several studies have compared OC with other NiTi files, all of which reported that OC files showed a higher cyclic fatigue resistance compared to other tested files [20-24], which is in agreement with the results of the present study.

Heat-treated files demonstrate a higher resistance to fatigue failure than the austenitic and $\mathrm{M}$-wire-based files [16]. PTG and OC files are both heat-treated; however, the latter is based on a $\mathrm{C}$-wire alloy which is a proprietary material, exclusively developed and implemented by Micro Mega, and files are said to have controlled memory and ability to prebend for easier access to the root canal along with elimination of constraints [19].

Cross-sectional geometry and center core area affect the flexibility of the rotary NiTi files $[2,14]$. A previous study found that files with high cross-sectional and center core areas are stiffer than files with other designs. Triangular cross sections resulted in increased flexibility than square designs at the same external taper [15]. OC files have a triangular cross section [19], while PTG files have a convex triangular cross section [17]; this increased mass in the center core area of the PTG files because the convex triangular design might explain the reduced fatigue resistance compared with OC files. In a recent study, the core diameter of OC files was measured and found to be very minimal (approximately $48,327 \mu \mathrm{m}^{2}$ ) compared to other tested files, which enhances its flexibility and subsequent fatigue resistance [22].

It has also been reported that files with larger taper have less resistance to fatigue failure than files with smaller taper
[15]. Although the tip sizes for both tested files, in the present study, are ISO 25, their tapers vary. The taper for PTG/F2 is 0.08 for the apical three millimeters, which progressively decreases until D16 [17], whereas OC files have a constant taper of 0.06 from the tip until D16 [19]. This might additionally justify the increased fatigue resistance of OC size 25 over PTG/F2 in the present study.

Additionally, it was specifically inferred, from the present study, that the cyclic fatigue resistance for both files was significantly decreased after 5 minutes of immersion in $\mathrm{NaOCl}$.

Several studies, with conflicting results, have been conducted to evaluate the effect on files immersed in $\mathrm{NaOCl}$. In a previous study, ProFile and $\mathrm{RaCe} \mathrm{NiTi}$ files size 25 taper 04 immersed in a heated $5.25 \% \mathrm{NaOCl}$ solution for 1 or 2 hours showed significant reduction in fatigue resistance by $40 \%$ or more [7]. Immersion of Reciproc R25 and WaveOne in $2.5 \% \mathrm{NaOCl}$ for $5 \mathrm{~min}$ decreased the cyclic fatigue resistance significantly $(p<0.05)$ as compared to the control group [10].

Palma et al. [12] concluded in their study that immersion in $3 \% \mathrm{NaOCl}$ solution for 1 or $5 \mathrm{~min}$ decreased the resistance to cyclic fatigue of ProTaper Next, Hyflex CM, and Hyflex EDM, with more significance shown for those manufactured from CM wire. A study in 2018 reported that the cyclic fatigue resistance of PTG files immersed in $\mathrm{NaOCl}$ dropped significantly $(p<0.05)$ when compared to those immersed in distilled water. The reduction in the resistance to cyclic fatigue when exposed to $\mathrm{NaOCl}$ can be attributed to the induced corrosive areas. It has been stated that active $\mathrm{ClO}^{-}$anions can increase corrosion [28]. Corrosion is sought to create selective removal of surface nickel with micropitting, which makes microstructural defects turn into cracks when submitted to stress, leading to a weakened instrument structure [3].

Although the abovementioned studies are in agreement with the results of the present study, the opposite has also been reported. A study found that static or dynamic immersion in 5\% $\mathrm{NaOCl}$ for 1 minute or 5 minutes does not significantly reduce the cyclic fatigue resistance of Twisted Files, Revo S SU files, and Mtwo files [3]. Similarly, another study concluded that reciprocating dynamic immersion in $5 \% \mathrm{NaOCl}$ for 1 or 5 minutes did not reduce significantly the cyclic fatigue resistance of One Shape, Reciproc R25, or WaveOne Primary [9]. Another study was conducted in 2018, which assessed the effects of sodium hypochlorite $(\mathrm{NaOCl})$ immersion and sterilization on the cyclic fatigue resistance of Twisted files and Hyflex CM. They 
concluded that the cyclic fatigue resistance of the two heattreated NiTi files tested was not significantly reduced following immersion in $\mathrm{NaOCl}$ [13].

The method used in this study for cyclic fatigue was based on a method described by Pruett et al. [29]. This method accurately describes the root canal curvature based on the angle of curvature and radius of curvature, and it provided the files with a suitable simulated root canal with a $60^{\circ}$ angle of curvature and $5 \mathrm{~mm}$ radius of curvature, measured according to the method of Schneider [30]. The artificial canal wall was flooded with synthetic oil to minimize the friction between the tested files and the simulated canal wall [31]. To remain within a realistic timeframe of clinical practice and considering the mean life of the control instruments, 1 minute and 5 minutes were selected as the contact time of the solution with the file.

The limitations in the study were that both the immersion in sodium hypochlorite and the cyclic fatigue test were done in a static position rather than a dynamic motion. A dynamic motion would mimic the clinical scenario more than a static position.

Within the limitations of this study, it can be concluded that OC files showed significantly more resistance to cyclic fatigue failure compared to PTG under the test conditions. Furthermore, immersion in $2.5 \% \mathrm{NaOCl}$ for 5 minutes significantly reduced the cyclic fatigue resistance for both the tested files.

\section{Data Availability}

The data used to support the findings of this study can be made available upon request to the corresponding author.

\section{Conflicts of Interest}

The authors declare that there are no conflicts of interest.

\section{Authors' Contributions}

Both authors have contributed significantly and agree with the manuscript.

\section{References}

[1] Y. Shen, H.-m. Zhou, Y.-f. Zheng, B. Peng, and M. Haapasalo, "Current challenges and concepts of the thermomechanical treatment of nickel-titanium instruments," Journal of Endodontics, vol. 39, no. 2, pp. 163-172, 2013.

[2] E. Sch€afer, A. Dzepina, and G. Danesh, "Bending properties of rotary nickel-titanium instruments," Oral Surgery, Oral Medicine, Oral Pathology, and Oral Radiology, vol. 96, pp. 757-763, 2003.

[3] E. Pedullà, N. M. Grande, G. Plotino, A. Pappalardo, and E. Rapisarda, "Cyclic fatigue resistance of three different nickel-titanium instruments after immersion in sodium hypochlorite," Journal of Endodontics, vol. 37, no. 8, pp. 1139-1142, 2011.

[4] P. Parashos and H. H. Messer, "Rotary NiTi instrument fracture and its consequences," Journal of Endodontics, vol. 32, no. 11, pp. 1031-1043, 2006.
[5] G. S. P. Cheung, B. Peng, Z. Bian, Y. Shen, and B. W. Darvell, "Defects in ProTaper S1 instruments after clinical use: fractographic examination," International Endodontic Journal, vol. 38, no. 11, pp. 802-809, 2005.

[6] C. E. Erik and T. Özyürek, "Effects of etidronate, $\mathrm{NaOCl}$, EDTA irrigation solutions and their combinations on cyclic fatigue resistance of nickel-titanium single-file rotary and reciprocating instruments at body temperature," Odontology, vol. 107, no. 2, pp. 190-195, 2019.

[7] O. A. Peters, J. O. Roehlike, and M. A. Baumann, "Effect of immersion in sodium hypochlorite on torque and fatigue resistance of nickel-titanium instruments," Journal of Endodontics, vol. 33, no. 5, pp. 589-593, 2007.

[8] E. Pedullà, N. M. Grande, G. Plotino, F. Palermo, G. Gambarini, and E. Rapisarda, "Cyclic fatigue resistance of two reciprocating nickel-titanium instruments after immersion in sodium hypochlorite," International Endodontic Journal, vol. 46, no. 2, pp. 155-159, 2013.

[9] A. Dagna, R. Beltrami, M. Colombo, M. Chiesa, S. Bianchi, and C. Poggio, "Cyclic fatigue resistance of three single-use NiTi instruments after immersion in sodium hypochlorite," International Journal of Experimental Dental Science, vol. 3, no. 2, pp. 67-72, 2014.

[10] C. Champa, V. Divya, A. Srirekha, R. Karale, A. Shetty, and P. Sadashiva, "An analysis of cyclic fatigue resistance of reciprocating instruments in different canal curvatures after immersion in sodium hypochlorite and autoclaving: an in vitro study," J Conserv Dent, vol. 20, pp. 194-198, 2017.

[11] H. Alfawaz, A. Alqedairi, H. Alsharekh, E. Almuzaini, S. Alzahrani, and A. Jamleh, "Effects of sodium hypochlorite concentration and temperature on the cyclic fatigue resistance of heat-treated nickel-titanium rotary instruments," Journal of Endodontics, vol. 44, no. 10, pp. 1563-1566, 2018.

[12] P. J. Palma, A. Messias, A. R. Cerqueira et al., "Cyclic fatigue resistance of three rotary file systems in a dynamic model after immersion in sodium hypochlorite," Odontology, vol. 107, no. 3, pp. 324-332, 2018.

[13] E. Pedullà, A. Benites, G. M. La Rosa et al., "Cyclic fatigue resistance of heat-treated nickel-titanium instruments after immersion in sodium hypochlorite and/or sterilization," Journal of Endodontics, vol. 44, no. 4, pp. 648-653, 2018.

[14] X. Xu, M. Eng, Y. Zheng, and D. Eng, "Comparative study of torsional and bending properties for six models of nickeltitanium root canal instruments with different cross-sections," Journal of Endodontics, vol. 32, no. 4, pp. 372-375, 2006.

[15] A. Versluis, H.-C. Kim, W. Lee, B.-M. Kim, and C.-J. Lee, "Flexural stiffness and stresses in nickel-titanium rotary files for various pitch and cross-sectional geometries," Journal of Endodontics, vol. 38, no. 10, pp. 1399-1403, 2012.

[16] J. Zupanc, N. Vahdat-Pajouh, and E. Schäfer, "New thermomechanically treated NiTi alloys - a review," International Endodontic Journal, vol. 51, no. 10, pp. 1088-1103, 2018.

[17] Dentsply ProTaper, Gold Rotary Files Brochure, Dentsply ProTaper, Charlotte, NC, USA, 2014, https://www.dentsply. com/content/dam/dentsply/pim/manufacturer/Endodontics/ Glide_Path_Shaping/Rotary_Reciprocating_Files/Shaping/ ProTaper_Gold_Rotary_Files/ProTaper-Gold-Brochure-p7btc wy-en-1502.pdf.

[18] A. Hieawy, M. Haapasalo, H. Zhou, Z.-j. Wang, and Y. Shen, "Phase transformation behavior and resistance to bending and cyclic fatigue of ProTaper Gold and ProTaper Universal Instruments," Journal of Endodontics, vol. 41, no. 7, pp. 1134-1138, 2015. 
[19] Micro-Mega, One Curve Files Brochure, Micro-Mega, Besançon, France, 2018, http://micromega.com/wp-content/ uploads/2018/03/Brochure-One-Curve-EN-1.pdf.

[20] İF. Ertuğrul, "Effect of sodium hypochlorite on the cyclic fatigue resistance: a scanning electron microscopy evaluation," Microscopy Research and Technique, vol. 82, no. 12, pp. 2089-2094, 2019.

[21] H. S. Topçuoğlu, G. Topçuoğlu, Ö Kafdağ, and H. Balkaya, "Effect of two different temperatures on resistance to cyclic fatigue of one Curve, EdgeFile, HyFlex CM and ProTaper next files," Australian Endodontic Journal: The Journal of the Australian Society of Endodontology Inc, vol. 46, no. 1, pp. $68-72,2020$.

[22] A. M. Elnaghy and S. E. Elsaka, "Cyclic fatigue resistance of one Curve, 2Shape, ProFile vortex, vortex blue, and RaCe nickel-titanium rotary instruments in single and double curvature canals," Journal of Endodontics, vol. 44, no. 11, pp. 1725-1730, 2018.

[23] M. Serafin, M. De Biasi, V. Franco, and D. Angerame, "In vitro comparison of cyclic fatigue resistance of two rotary single-file endodontic systems: OneCurve versus OneShape," Odontology, vol. 107, no. 2, pp. 196-201, 2019.

[24] S. Taffoli, N. M. Grande, G. Plotino et al., "Influence of environmental temperature, heat-treatment and design on the cyclic fatigue resistance of three generations of a single-file nickel-titanium rotary instrument," Odontology, vol. 107, pp. 301-307, 2019.

[25] C. M. Larsen, I. Watanabe, G. N. Glickman, and J. He, “Cyclic fatigue analysis of a new generation of nickel titanium rotary instruments," Journal of Endodontics, vol. 35, no. 3, pp. 401-403, 2009.

[26] Y. Haïkel, R. Serfaty, G. Bateman, B. Senger, and C. Allemann, "Dynamic and cyclic fatigue of engine-driven rotary nickeltitanium endodontic instruments," Journal of Endodontics, vol. 25, no. 6, pp. 434-440, 1999.

[27] A. Jamleh, Y. Yahata, A. Ebihara, A. R. Atmeh, T. Bakhsh, and H. Suda, "Performance of NiTi endodontic instrument under different temperatures," Odontology, vol. 104, no. 3, pp. 324-328, 2016.

[28] D. Sonntag and K. Heithecker, "Korrosion von Nickel-titaninstrumenten," Endodontie, vol. 15, pp. 23-30, 2006.

[29] J. P. Pruett, D. J. Clement, and D. L. Carnes, "Cyclic fatigue testing of nickel-titanium endodontic instruments," Journal of Endodontics, vol. 23, no. 2, pp. 77-85, 1997.

[30] S. W. Schneider, "A comparison of canal preparations in straight and curved root canals," Oral Surgery, Oral Medicine, Oral Pathology, vol. 32, no. 2, pp. 271-275, 1971.

[31] H. H. Nguyen, H. Fong, A. Paranjpe, N. M. Flake, J. D. Johnson, and O. A. Peters, "Evaluation of the resistance to cyclic fatigue among protaper next, ProTaper universal, and vortex blue rotary instruments," Journal of Endodontics, vol. 40, no. 8, pp. 1190-1193, 2014. 